\title{
1 Structural health monitoring with non-linear sensor measurements robust to unknown non-stationary input forcing
}

4

\author{
Subhamoy Sen ${ }^{\mathrm{a}, *}$, Neha Aswal ${ }^{\mathrm{a}}$, Qinghua Zhang ${ }^{\mathrm{b}}$, Laurent $_{\text {Mevel }}{ }^{\mathrm{b}}$ \\ ${ }^{a}$ Indian Institute of Technology Mandi, Mandi, HP, India \\ ${ }^{b}$ Univ. Gustave Eiffel, Inria, COSYS-SII, I4S Team, France
}

\section{Abstract}

7 Bayesian filtering based structural health monitoring algorithms typically assume stationary white Gaussian

8 noise models to represent an unknown input forcing. However, typical structural damages occur mostly under

9 the action of extreme loading conditions, like earthquake or high wind/waves, which are characteristically

10 non-stationary and non-Gaussian. Clearly, this invalidates this basic assumption, causing these algorithms to

11 perform poorly under non-stationary noise conditions. This paper extends an existing interacting filtering 
For dynamics of mechanical structure, idealized as a Markov process and defined in state-space form with state variables $\mathbf{x}_{1: k}$ observed for a time span of $[1: k]$ through a measurement sequence $\mathbf{y}_{1: k}$, Bayesian filtering employs two probabilistic models: i) for system state propagation $\mathbf{p}\left(\mathbf{x}_{k} \mid \mathbf{x}_{k-1}\right)$ formulated by the Chapman-Kolmogorov equation as $\mathbf{p}\left(\mathbf{x}_{k}, \mathbf{x}_{k-1}\right)=\mathbf{p}\left(\mathbf{x}_{k-1}\right) \times \mathbf{p}\left(\mathbf{x}_{k} \mid \mathbf{x}_{k-1}\right)$ and, ii) likelihood estimation model of states, i.e., $\mathbf{p}\left(\mathbf{y}_{k} \mid \mathbf{x}_{k}\right)$ for state estimate correction, to estimate recursively the states using the measurement sequence $\mathbf{y}_{1: k}$. The sequence $\mathbf{y}_{1: k}$ can be linear, non-linear or mixed structural response through which the structural health, parameterized with a set of location-based structural health indices (HIs), can be interpreted. Eventually, SHM with Bayesian filtering is posed as a joint probability estimation of state and parameters to obtain $\mathbf{p}\left(\mathbf{x}_{k}, \boldsymbol{\theta}_{k} \mid \mathbf{y}_{1: k}\right)$, in order to detect, localize and quantify the damages; where the additional states, $\boldsymbol{\theta}_{k}$, signify the $\mathbf{H I}$ s.

There exist successful applications of Bayesian filtering in SHM wherein HIs in $\boldsymbol{\theta}_{k}$ are tracked to localize any deterioration in structural health [31, 36, 38, 42. In the related literature, $\boldsymbol{\theta}_{k} \mathrm{~s}$ are mostly augmented in the state definition as $\mathbf{X}_{k}=\left[\mathbf{x}_{k} ; \boldsymbol{\theta}_{k}\right]$ to estimate them alongside $\mathbf{x}_{k}$ [5, 9-11]. Yet, owing to the induced non-linearity and/or the loss of observability, this approach is reported to cause divergence, leading to false or infeasible solutions, especially for time varying systems [11. Recently, an interacting filtering strategy has emerged as a reliable alternative to tackle time varying systems with moderate state size. With this approach, a conditional posterior distribution estimation for the system states is followed by a marginal posterior distribution of the system parameters (also known as Rao-Blackwellisation) [4, 13, 35, 38]. The advantage of the interacting approach, in terms of computational burden and stability, over the joint estimation approach has been discussed in [8].

Nevertheless, the likelihood estimation function, $\mathbf{p}\left(\mathbf{y}_{k} \mid \boldsymbol{\theta}_{k}\right)$, for parameter estimation problems, is typically a non-linear mapping of $\mathbf{x}_{k} / \boldsymbol{\theta}_{k}$ (e.g., finite element (FE) models) for which only non-linear filter variants (e.g., Extended (EKF) [21, Unscented (UKF) [23, 30, Ensemble Kalman filter (EnKF), Particle filter (PF), etc.) are applicable. PF [18] has been recognized as a powerful approach in this endeavor [2, 7, 9] with some concerns regarding its computational expense [39]. To manage the computational expense, the Interacting Particle-Kalman filter (IPKF) [36, 38, was introduced, that efficiently handles the linear state estimation with linear measurements (e.g. acceleration, displacement, etc.) using a standard KF while PF handles the non-linear parameter estimation. However, because of the use of KF, the applicability of IPKF is limited to linear systems (linear state propagation and measurements) only.

To generalize the application of IPKF to non-linear systems, KF therefore should be replaced with nonlinear filter variants like EKF/UKF/EnKF. As an alternative, EKF employs approximate local linearization through first order Taylor approximation. The associated Jacobian calculation, even being computeintensive, does not usually hinder estimation for moderate sized systems. Nevertheless, some comparative studies [19, 20] identified that EKF's approximate closure scheme may lack accuracy and hamper the detection promptness. With UKF, on the other hand, uncertainty propagation through a sparse set of "sigma 
points" limits its performance for severely non-linear systems [20, 40. EnKF employs a set of ensembles realized from the entire domain of states - for uncertainty propagation while preserving the non-linearity in state transition. It also offers flexibility to enhance the accuracy through the employment of bigger ensemble pools. The selection of EnKF in this study takes basis on the comparative study of [20].

Filtering based SHM techniques typically idealize the unknown input force as a Stationary White Gaussian Noise (SWGN). Contrarily, the exogenous forces, that can potentially damage civil infrastructures (e.g., seismic forcing or heavy wind/waves), are mostly unforeseen and do not satisfy this assumption. Clearly, to ensure estimation accuracy and subsequently the structural safety, the adopted SHM technique has to be robust against input forcing. 24] proposed an unbiased minimum-variance linear state estimation filter that does not require prior information about the unknown input. This was later improved for practical application by [22. Joint state and input estimation has been employed by [16] for a system without a direct transmission term, and later updated for a system with a direct transmission term [17. To avoid numerical instability especially for the systems that are redundantly instrumented, 26] proposed similar filters that jointly estimate states and inputs. However, it was mentioned that the unobservability in the system may lead to an estimation instability, for this augmentation strategy.

3] proposed a dual PF for exogenous force estimation for time invariant systems. [1] combined the parameters and the inputs together in a very large state vector in order to estimate the seismic excitation acting on a linear time invariant (LTI) system. The estimation of the input alongside the system matrices and states have been done by [38]. An analogous approach focusing on the estimation of the input statistics has been presented in [37. for systems with varying noise level. [15] presents an auto-covariance least square based method for estimating noise covariances online for linear time varying (LTV) as well as non-linear systems.

6] proposed an UKF based algorithm that employs a time varying auto-regressive model to jointly estimate the structural parameters and the unknown inputs. For time varying systems, 29] generalized their earlier proposal [28] of a smoothing algorithm for joint estimation of state, parameters and input. With a similar objective, [12 proposed a dual filtering approach in which the structural parameters, as augmented states, are jointly estimated with the response states conditioned on an estimate for the input force. Instead of an explicit reconstruction of the input forces, 34] estimated the input force model through the parameters of a Gaussian process within a Bayesian framework.

Recently, for LTV systems with known system matrices, instead of estimating the input time histories [12, 29, 38, or its statistics [34, [43] developed a robust and stable linear state estimator unaffected by unknown inputs. This unknown input rejection approach is used in Kalman filter environment with linear measurements. With an intent to generalize earlier works of IPKF 42] for non-linear systems with an added robustness against unknown external disturbances, PF has been coupled with EnKF. While the authors' previous works [38] approached this input robustness through an explicit estimation of inputs online, the 
present article rejects the effect of input variability through output injection for a modified state transition equation following 43 .

Rejection of unknown inputs is usually addressed by unknown input observers, with a rich literature on this topic (see 43 and references therein). While most unknown input observers are limited to linear systems, they do not consider parameter estimation in addition to state estimation. The methods for designing such unknown input observers cannot be applied to EnKF and PF. The method for unknown input rejection adopted in this paper is not limited to a particular unknown input observer design. It rejects unknown inputs by simply transforming the system model, so that state and/or parameter estimation algorithms can be applied as if the rejected unknown inputs no longer exist. This output injection has therefore been embedded within the EnKF environment to yield input-robust response state estimates for non-linear systems. In this process, the present article also overcomes the limitation of IPKF 38 of using linear measurements (e.g. acceleration) only, and extend its applicability to non-linear measurements (e.g. strain) as well. The novel contributions of the study can therefore be listed as: a novel noise robust Interacting Particle-Ensemble Kalman Filter (rIP-EnKF) algorithm in which, i) PF coupled with EnKF extends the reach of 38] to non-linear systems, ii) noise robust EnKF ensures rejection of unknown non-stationary excitation and finally, iii) linear and non-linear measurements can be dealt with, simultaneously.

The proposed algorithm is predictor model-based that involves a precise model (preferably a calibrated FE model) for state propagation. At least a few accelerometer measurement channels are assumed to be available for perfect functioning of the algorithm. Also, sensor noise statistics corresponding to accelerometers and strain gauges are assumed to be available. The system dynamics is assumed to remain linear even after damage.

It should be noted here, that stability of state estimation algorithms with unknown input rejection is studied in the literature of unknown input observers for LTI systems and for some affine parameter varying systems. To our knowledge, the only stability analysis of such algorithms for general LTV systems has been proposed in [43. The generalization of this analysis to EnKF with output injection, applied in this paper to address non-linearities, is certainly an important and difficult task clearly outside of the scope of this paper.

In the following, based on the non-linear state-space modeling detailed in Section 2, output injection is demonstrated in Section 3, followed by the detailed proposal in Section 4. The proposed approach is tested on a numerical simulation in Section 5.

\section{Modeling and system dynamics}

The stiffness and damping matrices of LTV mechanical system, i.e., $\mathbf{K}(\boldsymbol{\theta}(t))_{n \times n}$ and $\mathbf{C}(\boldsymbol{\theta}(t))_{n \times n}$, are functions of time varying location based HIs represented by the time varying parameter vector $\boldsymbol{\theta}(t)_{N_{\theta} \times 1}$. The dynamics of such a system under seismic excitation, in state space domain, can be described by a time invariant mass matrix $\mathbf{M}$, a time varying stiffness matrix $\mathbf{K}(\boldsymbol{\theta}(t))$ and damping matrix $\mathbf{C}(\boldsymbol{\theta}(t))$ [42. Defining 
${ }_{134} \quad \mathbf{B}_{c}=\left[\begin{array}{c}\mathbf{0}_{n \times m} \\ \mathbf{M}^{-1}\end{array}\right]_{2 n \times m}, \mathbf{E}_{c}=\left[\begin{array}{c}\mathbf{0}_{n \times r} \\ \tau\end{array}\right]_{2 n \times r}$ and $\mathbf{F}(t)=\left[\begin{array}{cc}\mathbf{0}_{n \times n} & \mathbf{I}_{n \times n} \\ -\mathbf{M}^{-1} \mathbf{K}(\boldsymbol{\theta}(t)) & -\mathbf{M}^{-1} \mathbf{C}(\boldsymbol{\theta}(t))\end{array}\right]_{2 n \times 2 n}$, the

$$
\dot{\mathbf{x}}(t)=\mathbf{F}(t) \mathbf{x}(t)+\mathbf{B}_{c} \mathbf{u}(t)+\mathbf{E}_{c} \ddot{\mathbf{a}}^{g}(t)
$$

where system state, $\mathbf{x}(t)=\left[\begin{array}{ll}\mathbf{q}(t) & \dot{\mathbf{q}}(t)\end{array}\right]_{2 n \times 1}^{T}$, with $\mathbf{q}(t)$ and $\dot{\mathbf{q}}(t)$ representing the displacement and velocity responses. $\mathbf{0}$ and $\mathbf{I}$ are null and identity matrices of mentioned dimensions, respectively. $\mathbf{u}(t)_{m \times 1}$ is encompassing both the process noise and the ambient force acting on the structure and will be collectively defined as process noise from now on. It is assumed that, $\mathbf{u}(t)_{m \times 1}$ has known statistics and can be modelled as an SWGN of constant covariance $\mathbf{Q}_{m \times m}$, which takes into account both ambient forces and model uncertainty. $\ddot{\mathbf{a}}^{g}(t)_{r \times 1}$ represents an unknown disturbance (e.g. seismic ground motion) which is an unknown arbitrary function of $t$, without any assumed statistical property. $r$ is the number of channels for the disturbance input.

The measurement $\mathbf{y}(t)$ can be a linear mapping (denoted here as linear measurement, $\mathbf{y}^{l}(t)$, e.g., relative acceleration, displacement, etc.) or a non-linear mapping (denoted here as non-linear measurement, $\mathbf{y}^{n l}(t)$, e.g., dynamic strain, etc.) of the state variable $\mathbf{x}(t)$. The adopted nomenclature for this measurement is in line with [25, 32, 33, 44]. A mix of $\mathbf{y}^{l}(t)$ or $\mathbf{y}^{n l}(t)$ is also possible. The present study adopts a combination of acceleration, as $\mathbf{y}^{l}(t)$, and strain, as $\mathbf{y}^{n l}(t)$, as measurements. The equation for the relative accelerations $\ddot{\mathbf{q}}_{p}(t)$, at $p$ accelerometers, can be written as,

$$
\ddot{\mathbf{q}}_{p}(t)=\mathfrak{L}[\mathbf{H}(t) \mathbf{x}(t)+\mathbf{D u}(t)]+\mathbf{L} \ddot{\mathbf{a}}^{g}(t)+\mathbf{w}^{a}(t)
$$

LTV measurement model, $\mathbf{H}(t)_{n \times 2 n}=\left[\begin{array}{ll}-\mathbf{M}^{-1} \mathbf{K}(\boldsymbol{\theta}(t)) & -\mathbf{M}^{-1} \mathbf{C}(\boldsymbol{\theta}(t))\end{array}\right]$ and LTI direct transmission matrix, $\mathbf{D}_{n \times m}=\mathbf{M}^{-1}$, maps $(2 n \times 1)$ order states and $(m \times 1)$ order inputs to the corresponding $n$ order acceleration response, $\ddot{\mathbf{q}}(t)$, at every dof (Degree of Freedom). $\mathbf{L}_{p \times r}$ maps the direct impact of $r$ disturbance channels to $p$ output channels. The location matrix $\mathfrak{L}_{p \times n}$ selects $p$ measured dofs from $n$. $\mathbf{w}^{a}(t)_{p \times 1}$, representing the measurement noise in $p$ accelerometers, is an SWGN process of covariance $\mathbf{R}_{p \times p}^{a}$.

In practice, for a base excited structure fitted with accelerometers patched onto its surface, the relative acceleration, $\ddot{\mathbf{q}}(t) / \ddot{\mathbf{q}}_{p}(t)$, can never be measured due to lack of fixed reference. Clearly, for such cases, the measured acceleration $\mathbf{y}^{l}(t)$ is a summation of $\ddot{\mathbf{q}}_{p}(t)$ with a contribution from the base acceleration $\ddot{\mathbf{a}}^{g}(t)$. The measurement equation, with respect to measured acceleration, $\mathbf{y}^{l}(t)$, can be presented as,

$$
\mathbf{y}^{l}(t)=\ddot{\mathbf{q}}_{p}(t)-\mathbf{L} \ddot{\mathbf{a}}^{g}(t)=\mathfrak{L}\{\mathbf{H}(t) \mathbf{x}(t)+\mathbf{D u}(t)\}+\mathbf{w}^{a}(t)
$$

Sampled at discrete time instants indexed by $k=0,1,2, \ldots$, Equations $(1)$ and $(3)$ then lead to the discrete 
time state-space model [1],

$$
\begin{array}{r}
\mathbf{x}_{k}=\mathbf{F}_{k} \mathbf{x}_{k-1}+\mathbf{B}_{k} \mathbf{u}_{k}+\mathbf{E}_{k} \ddot{\mathbf{a}}_{k}^{g} ; \\
\mathbf{y}_{k}^{l}=\mathbf{H}_{k} \mathbf{x}_{k}+\mathbf{D}_{k} \mathbf{u}_{k}+\mathbf{w}_{k}^{a}
\end{array}
$$

where $\mathbf{F}_{k}, \mathbf{B}_{k}, \mathbf{D}_{k}, \mathbf{E}_{k}, \mathbf{H}_{k}, \mathbf{x}_{k}, \mathbf{y}_{k}^{l}$ and $\mathbf{w}_{k}^{a}$ are the discrete time counterparts of the corresponding continuous time entities described above, obtained through zero-order-hold technique. The location matrix $\mathfrak{L}$ has been incorporated into $\mathbf{H}_{k}, \mathbf{D}_{k}$ and $\mathbf{w}_{k}^{a}$ for simplicity.

Following the time indexing scheme employed in [41, the inputs $\mathbf{u}_{k}$ and $\ddot{\mathbf{a}}_{k}^{g}$, sampled at the $(k-1)^{t h}$ time instant, take part in state transition from $\mathbf{x}_{k-1}$ (past) to $\mathbf{x}_{k}$ (current) which are observed at the current time instant as $\mathbf{y}_{k}^{l}$. $\mathbf{F}_{k}$ and $\mathbf{H}_{k}$, defined at the current time instant, are functions of $\boldsymbol{\theta}_{k}$ that remain constant over the time interval $k-1$ to $k$. The process uncertainty is carried over to the $k^{\text {th }}$ instant and eventually $\mathbf{y}_{k}^{l}$ is contaminated with $\mathbf{w}_{k}^{a}$ at the $k^{\text {th }}$ instant. The same time index formalism is also used in [38.

Strain measurements are additionally included in this study for system health estimation. The motivation behind this consideration is the relatively low cost of strain gauges over accelerometers. Discrete time strain response, $\varepsilon_{k}^{x x}$, denoted as discrete non-linear measurement $\mathbf{y}_{k}^{n l}$, measured at $s$ strain gauges, can be obtained by mapping the nodal displacements, $\mathbf{q}_{k}$ (a subset of $\mathbf{x}_{k}$ ) through the non-linear strain-displacement relationship $\mathbf{y}_{k}^{n l}=f\left(\mathbf{x}_{k}\right)+\mathbf{w}_{k}^{g}$; with $\mathbf{w}_{k}^{g}$ being the sensor noise in strain gauges, modelled as an SWGN of covariance $\mathbf{R}_{s \times s}^{g}$. A specific case of strain-displacement function for an Euler-Bernoulli beam has been demonstrated in Appendix A.

The strain response $\varepsilon_{k}^{x x}$ is then added into the state vector in order to estimate them alongside $\mathbf{x}_{k}$ resulting in an extended state vector, $\mathbf{X}_{k}=\left[\begin{array}{ll}\mathbf{x}_{k} & \varepsilon_{k-1}^{x x}\end{array}\right]^{T}$. With a non-linear state mutation function $\tilde{F}_{k}\left(\mathbf{X}_{k-1}\right)=\left[\begin{array}{l}\mathbf{F}_{k} \mathbf{x}_{k-1} \\ f\left(\mathbf{x}_{k-1}\right)\end{array}\right]$, where $f(\bullet)$ is the non-linear strain-displacement mapping mentioned earlier, the process and measurement equations can be redefined as,

$$
\begin{array}{r}
\mathbf{X}_{k}=\tilde{F}_{k}\left(\mathbf{X}_{k-1}\right)+\tilde{B}_{k} \mathbf{U}_{k}+\tilde{E}_{k} \mathcal{S}_{k}^{g} \\
\mathbf{Y}_{k}=\tilde{H}_{k} \mathbf{X}_{k}+\tilde{D}_{k} \mathbf{U}_{k}+\mathbf{W}_{k}
\end{array}
$$

180 where the linear system matrices have been defined as $\tilde{B}_{k}=\left[\begin{array}{c}\mathbf{B}_{k} \\ 0\end{array}\right], \tilde{E}_{k}=\left[\begin{array}{c}\mathbf{E}_{k} \\ 0\end{array}\right], \tilde{H}_{k}=\left[\begin{array}{cc}\mathbf{H}_{k} & \mathbf{0}_{p \times s} \\ \mathbf{0}_{s \times 2 n} \mathbf{I}_{s \times s}\end{array}\right]$, ${ }_{181} \quad \tilde{D}_{k}=\left[\begin{array}{c}\mathbf{D}_{k} \\ 0\end{array}\right]$, and the random processes as $\mathbf{Y}_{k}=\left[\begin{array}{c}\mathbf{y}_{k}^{l} \\ \mathbf{y}_{k}^{n l}\end{array}\right], \mathbf{U}_{k}=\left[\begin{array}{c}\mathbf{u}_{k} \\ 0\end{array}\right], \mathcal{S}_{k}^{g}=\left[\begin{array}{c}\ddot{\mathbf{a}}_{k}^{g} \\ 0\end{array}\right], \mathbf{W}_{k}=\left[\begin{array}{c}\mathbf{w}_{k}^{a} \\ \mathbf{w}_{k}^{g}\end{array}\right]$. 


\section{Unknown input rejection from system dynamics}

While the process noise $\mathbf{U}_{k}$ can be well modeled as a random noise, typically with an assumed Gaussian distribution, the unknown input $\mathcal{S}_{k}^{g}$ (e.g. seismic excitation) is totally arbitrary, without any prior statistical property, and not necessarily random. A major goal of this paper is to design an SHM approach robust to the unknown input $\mathcal{S}_{k}^{g}$ without assuming or estimating its statistical properties. This study thus neither reconstructs the unknown input $\mathcal{S}_{k}^{g}$ as in [12, 26, 27, 29, 38, nor estimates its statistics as in [15, 34]. Following the ideas of [43], the proposed approach ensures robustness against the unknown input $\mathcal{S}_{k}^{g}$ by rejecting its effect from the system dynamics by means of an output injection, as demonstrated in the following.

Owing to the output equation in (5), the following holds true with an arbitrary bounded matrix $\mathbf{G}_{k} \in$ $\mathbb{R}^{2 n+s \times p+s}$ :

$$
0=\mathbf{G}_{k}\left(\mathbf{Y}_{k}-\tilde{H}_{k} \mathbf{X}_{k}-\tilde{D}_{k} \mathbf{U}_{k}-\mathbf{W}_{k}\right)
$$

Setting,

$$
\mathcal{L}_{k}=\mathbf{I}_{2 n+s}-\mathbf{G}_{k} \tilde{H}_{k},
$$

the state equation in (5) is then rewritten as

$$
\begin{aligned}
\mathbf{X}_{k} & =\tilde{F}_{k}\left(\mathbf{X}_{k-1}\right)+\tilde{B}_{k} \mathbf{U}_{k}+\tilde{E}_{k} \mathcal{S}_{k}^{g}+\mathbf{G}_{k}\left(\mathbf{Y}_{k}-\tilde{H}_{k} \mathbf{X}_{k}-\tilde{D}_{k} \mathbf{U}_{k}-\mathbf{W}_{k}\right) \\
& =\mathcal{L}_{k} \tilde{F}_{k}\left(\mathbf{X}_{k-1}\right)+\mathcal{L}_{k} \tilde{E}_{k} \mathcal{S}_{k}^{g}+\mathcal{L}_{k} \tilde{B}_{k} \mathbf{U}_{k}+\mathbf{G}_{k}\left(\mathbf{Y}_{k}-\tilde{D}_{k} \mathbf{U}_{k}-\mathbf{W}_{k}\right)
\end{aligned}
$$

By choosing $\mathbf{G}_{k}$ so that the matrix $\mathcal{L}_{k}$ defined in $(7)$ satisfies,

$$
\mathcal{L}_{k} \tilde{E}_{k}=0
$$

Equation (8) gets decoupled from $\mathcal{S}_{k}^{g}$ and can be rewritten as,

$$
\mathbf{X}_{k}=\mathcal{F}_{k}\left(\mathbf{X}_{k-1}\right)+\mathcal{B}_{k} \mathbf{U}_{k}+\mathbf{G}_{k} \mathbf{Y}_{k}+\mathbf{V}_{k}
$$

with $\mathcal{F}_{k}(X)=\mathcal{L}_{k} \tilde{F}_{k}(X), \mathcal{B}_{k}=\mathcal{L}_{k} \tilde{B}_{k}-\mathbf{G}_{k} \tilde{D}_{k}$ and $\mathbf{V}_{k}=-\mathbf{G}_{k} \mathbf{W}_{k}$ modelled as an SWGN process of variance $\mathbf{G}_{k}\left[\begin{array}{rr}\mathbf{R}^{a} & \mathbf{0} \\ \mathbf{0} & \mathbf{R}^{g}\end{array}\right] \mathbf{G}_{k}^{T}$. The unknown input $\mathcal{S}_{k}^{g}$ has disappeared from Equation 100 , owing to an appropriate injection of the known output $\mathbf{Y}_{k}$ through $\mathbf{G}_{k}$. For Equation $\left(9\right.$ to be valid, $\mathbf{G}_{k}$ is chosen as: $\mathbf{G}_{k}=$ $\tilde{E}_{k}\left(\tilde{H}_{k} \tilde{E}_{k}\right)^{\dagger}$, where $\dagger$ denotes Moore-Penrose Pseudo-inverse operation. It is assumed that the inverse of the square matrix $\tilde{E}_{k}^{T} \tilde{H}_{k}^{T} \tilde{H}_{k} \tilde{E}_{k}$ exists and is bounded, so that the Penrose Pseudo-inverse of $\tilde{H}_{k} \tilde{E}_{k}$ is upper 
bounded. In the particular case of time invariant matrix product $\tilde{H} \tilde{E}$, it is sufficient that $\tilde{H} \tilde{E}$ is full column rank. This assumption implies that $p \geq r$.

Notice that when no linear measurements are available (i.e., $p=0$ ), Equation (6) yields $\mathbf{G}_{k}=0$, thus input rejection can not be achieved. With linear measurements only, rejecting $\mathcal{S}_{k}^{g}$ is possible following the lines of [43. With both linear/non-linear measurements, as in the current state transition function in (10), the rejection depends on the available number of linear measurements only with a condition of $p \geq r$ to ensure perfect robustness. Yet, due to the presence of non-linear measurements, the approach has to be modified since standard KF is no longer an option. Eventually, the part of Equation (10) (excluding the strain states) responsible for input rejection can be isolated as,

$$
\mathbf{x}_{k}=\overline{\mathbf{F}}_{k} \mathbf{x}_{k-1}+\overline{\mathbf{B}}_{k} \mathbf{u}_{k}+\mathbf{G}_{k}^{x} \mathbf{y}_{k}+\overline{\mathbf{v}}_{k}
$$

where, $\overline{\mathbf{F}}_{k}=\left(\mathbf{I}_{2 n}-\mathbf{G}_{k}^{x} \mathbf{H}_{k}\right) \mathbf{F}_{k}, \overline{\mathbf{B}}_{k}=\left(\mathbf{I}_{2 n}-\mathbf{G}_{k}^{x} \mathbf{H}_{k}\right) \mathbf{B}_{k}-\mathbf{G}_{k}^{x} \mathbf{D}_{k}$ and $\overline{\mathbf{v}}_{k}=-\mathbf{G}_{k}^{x} \mathbf{w}_{k}^{a}$. The new process noise $\overline{\mathbf{v}}_{k}$ in Equation (11) can still be defined as SWGN with an altered co-variance $\mathbf{G}_{k}^{x} \mathbf{R}_{k}^{a} \mathbf{G}_{k}^{x T}$, where $\left(\mathbf{I}_{2 n}-\mathbf{G}_{k}^{x} \mathbf{H}_{k}\right) \mathbf{E}_{k}=0$. Since the strain response states are non-linear explicit functions of the state subset $\mathbf{x}_{k}$, the induced robustness in $\mathbf{x}_{k}$ will also ensure robustness in the strain response states. Notice that any linear or non-linear measurement can be used in place of accelerations or strains respectively. This paper thus extends the assumptions of 5 where only linear measurements are considered.

\section{Robust Interacting Particle-Ensemble Kalman Filtering}

To generalize the IPKF methods [37, 38] to non-linear systems, the present work replaces KF within IPKF with EnKF to handle the non-linear state estimation problem as in Equation (5). In the modified interacting particle-ensemble Kalman filter (IP-EnKF), a set of EnKFs runs within an envelop of PF. Within PF, each particle represents a parameter instance that defines the structural matrices to be used in the process equation of the EnKF. Thus, both filters interact to obtain the conditional estimates for the response states and parameters simultaneously.

\subsection{Envelop Parameter Filter}

Bayesian belief propagation requires an explicit analytical integration to be performed over the entire state domain. This task is simple with Gaussian states evolving through a linear state transition. The current parameter estimation problem is, however, non-linear for which an explicit analytical integration over the entire parameter space is not possible. PF attempts a particle approximation of this integration by representing and propagating the parametric uncertainty through a cloud of $N_{p}$ independent particles $\boldsymbol{\Xi}_{k}=\left[\boldsymbol{\xi}_{k}^{1}, \boldsymbol{\xi}_{k}^{2}, \cdots, \boldsymbol{\xi}_{k}^{N_{p}}\right]$ listing all individual particles as $\boldsymbol{\xi}_{k N_{\theta} \times 1}^{i}$, where $N_{\theta}$ denotes the number of health parameters $(\mathbf{H I})$ that are to be estimated. Additionally, no presumption on the stochastic nature of the 
parameter states is enforced. The time evolution of these particles is a random perturbation around their current position $\boldsymbol{\xi}_{k-1}^{j}$ along with a forced shift towards the current particle mean estimate $\overline{\boldsymbol{\xi}}_{k-1}[38$,

$$
\boldsymbol{\xi}_{k}^{j}=\alpha \boldsymbol{\xi}_{k-1}^{j}+\mathbb{N}\left(\delta \boldsymbol{\xi}_{k} ; \boldsymbol{\sigma}_{k}^{\boldsymbol{\xi}}\right)
$$

where a Gaussian blurring is performed on $\boldsymbol{\xi}_{k-1}^{j}$ with a shift $\delta \boldsymbol{\xi}_{k}=(1-\alpha) \overline{\boldsymbol{\xi}}_{k-1}$ and a spread of $\boldsymbol{\sigma}_{k}^{\xi}$, $\alpha$ is a hyper-parameter that controls the turbulence in the estimation. The evolved particles are assigned a weight which gets updated on each iteration based on their likelihood, detailed later in this article.

\subsection{Nested robust state filter}

Unlike the typical EnKF formalism, the EnKF in the present study does not model the unknown input disturbance, $\mathcal{S}_{k}^{g}$, as a case of SWGN. Instead, rejection of this unknown input through output injection is approached (as detailed in Section 3) to gain robustness against $\mathcal{S}_{k}^{g}$. To accommodate this modification, the state and measurement equations within EnKF have been redefined accordingly.

For any arbitrary $j^{\text {th }}$ particle $\boldsymbol{\xi}_{k}^{j}$ among the $N_{p}$ parameter particles available at the $k^{\text {th }}$ time step, $N_{e}$ state ensemble $\left(\left\{\mathbf{X}_{k \mid k}^{i, j}\right\} ; \quad i=1,2, \cdots, N_{e}\right)$ are propagated over time using EnKF. Using a set of simulated SWGN processes $\mathbf{U}_{k}^{i, j}, \mathbf{V}_{k}^{i, j}$ and $\mathbf{W}_{k}^{i, j}$, with variances as defined in Section 3 the $i^{t h}$ ensemble is propagated and subsequently observed as follows as per Equations (5) and (10),

$$
\begin{array}{r}
\mathbf{X}_{k \mid k-1}^{i, j}=\mathcal{F}_{k}^{j}\left(\mathbf{X}_{k-1 \mid k-1}^{i, j}\right)+\mathcal{B}_{k}^{j} \mathbf{U}_{k}^{i, j}+\mathbf{G}_{k}^{j} \mathbf{Y}_{k}+\mathbf{V}_{k}^{i, j} \\
\mathbf{Y}_{k \mid k-1}^{i, j}=\tilde{H}_{k}^{j} \mathbf{X}_{k \mid k-1}^{i, j}+\tilde{D}_{k}^{j} \mathbf{U}_{k}^{i, j}+\mathbf{W}_{k}^{i, j}
\end{array}
$$

The corresponding innovation for each of the $i^{\text {th }}$ ensemble can be obtained as a departure of predicted measurement $\mathbf{Y}_{k \mid k-1}^{i, j}$ from the actual measurement $\mathbf{Y}_{k}$ as $\boldsymbol{\epsilon}_{k \mid k-1}^{i, j}=\mathbf{Y}_{k}-\mathbf{Y}_{k \mid k-1}^{i, j}$, with an ensemble mean $\epsilon_{k \mid k-1}^{j}=\frac{1}{N_{e}} \sum_{i=1}^{N_{e}} \epsilon_{k \mid k-1}^{i, j}$. The cross-covariance between the state and the measurement prediction $\boldsymbol{C}_{k}^{j, X Y}$ and the innovation covariance $\boldsymbol{S}_{k}^{j}$ can be computed as suggested in [14,

$$
\begin{aligned}
\boldsymbol{C}_{k}^{j, \mathbf{X Y}} & =\frac{1}{N_{e}-1} \sum_{i=1}^{N_{e}}\left\{\mathbf{X}_{k \mid k-1}^{j}-\mathbf{X}_{k \mid k-1}^{i, j}\right\}\left\{\mathbf{Y}_{k \mid k-1}^{j}-\mathbf{Y}_{k \mid k-1}^{i, j}\right\}^{T} \\
\boldsymbol{S}_{k}^{j}=\boldsymbol{C}_{k}^{j, \mathbf{Y Y}} & =\frac{1}{N_{e}-1} \sum_{i=1}^{N_{e}}\left\{\mathbf{Y}_{k \mid k-1}^{j}-\mathbf{Y}_{k \mid k-1}^{i, j}\right\}\left\{\mathbf{Y}_{k \mid k-1}^{j}-\mathbf{Y}_{k \mid k-1}^{i, j}\right\}^{T}
\end{aligned}
$$

where $\mathbf{X}_{k \mid k-1}^{j}$ and $\mathbf{Y}_{k \mid k-1}^{j}$ are the ensemble mean estimates for the propagated states and predicted measurements, which can be obtained as: $\mathbf{X}_{k \mid k-1}^{j}=\frac{1}{N_{e}} \sum_{i=1}^{N_{e}} \mathbf{X}_{k \mid k-1}^{i, j}$ and $\mathbf{Y}_{k \mid k-1}^{j}=\frac{1}{N_{e}} \sum_{i=1}^{N_{e}} \mathbf{Y}_{k \mid k-1}^{i, j}$. The EnKF gain can further be obtained as $\mathbf{K}_{k}^{j}=C_{k}^{j, \mathbf{X Y}}\left(\mathbf{S}_{k}^{j}\right)^{-1}$. Finally, the state ensembles are updated as,

$$
\mathbf{X}_{k \mid k}^{i, j}=\mathbf{X}_{k \mid k-1}^{i, j}+\mathbf{K}_{k}^{j} \epsilon_{k \mid k-1}^{i, j}
$$

\footnotetext{
${ }^{1} A+B \mathbb{N}(\mu ; \sigma)$ means $A+B z$ where $z$ follows $\mathbb{N}(\mu ; \sigma)$
} 
leading to the particle approximation for state and parameter estimates as,

$$
\mathbf{X}_{k \mid k}=\sum_{j=1}^{N_{p}} w\left(\boldsymbol{\xi}_{k}^{j}\right) \mathbf{X}_{k \mid k}^{j} \text { and } \overline{\boldsymbol{\xi}}_{k}=\sum_{j=1}^{N_{p}} w\left(\boldsymbol{\xi}_{k}^{j}\right) \boldsymbol{\xi}_{k}^{j} .
$$

Thus with EnKF, a set of prior state ensembles, i.e., $\left\{\mathbf{X}_{k-1 \mid k-1}^{i, j}\right\}$ gets updated as $\left\{\mathbf{X}_{k \mid k}^{i, j}\right\}$ with the ensemble mean $\mathbf{X}_{k \mid k}^{j}$ without being affected by $\mathcal{S}_{k}^{g}$.

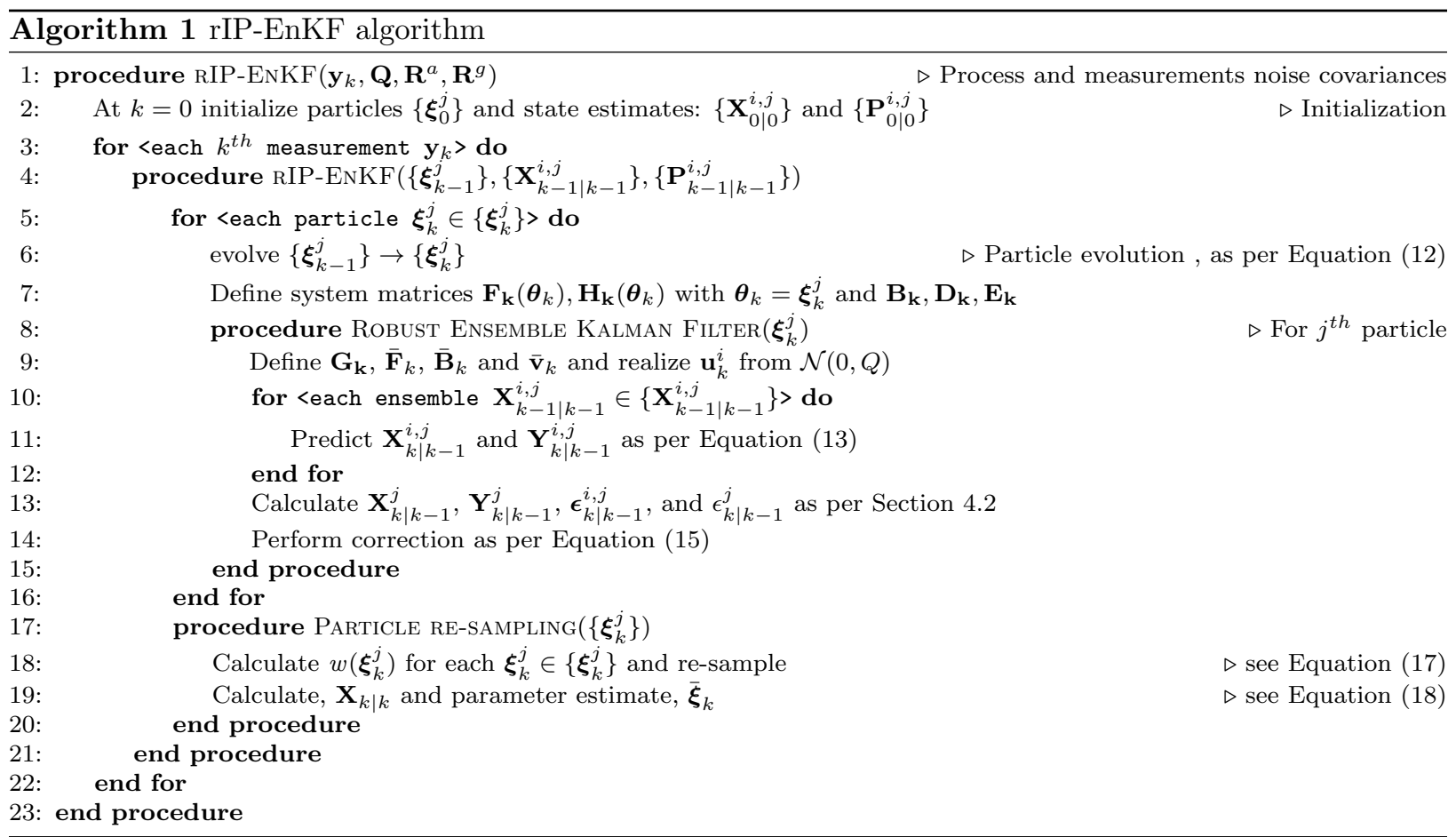

\subsection{Particle approximation}

Since $\mathbf{X}_{k \mid k}^{j}$ is conditioned on particle $\boldsymbol{\xi}_{k}^{j}$, the likelihood of $\boldsymbol{\xi}_{k}^{j}$, i.e., $\mathcal{L}\left(\boldsymbol{\xi}_{k}^{j}\right)=\mathbf{p}\left(\mathbf{Y}_{k} \mid \boldsymbol{\theta}_{k}=\boldsymbol{\xi}_{k}^{j}\right)$ can thus be defined using the ensemble mean of innovation, $\epsilon_{k \mid k-1}^{j}$, and the error covariance, $\mathbf{S}_{k}^{j}$, as,

$$
\mathcal{L}\left(\boldsymbol{\xi}_{k}^{j}\right)=\mathbf{p}\left(\mathbf{Y}_{k} \mid \boldsymbol{\theta}_{k}=\boldsymbol{\xi}_{k}^{j}\right)=(2 \pi)^{-(p+s) / 2}\left|\mathbf{S}_{k}^{j}\right|^{-1 / 2} \exp ^{-\frac{1}{2} \epsilon_{k \mid k-1}^{j}{ }^{T} \mathbf{S}_{k}^{j-1} \epsilon_{k \mid k-1}^{j}}
$$

Using $\mathcal{L}\left(\boldsymbol{\xi}_{k}^{j}\right)$, the normalized updated weight for $\boldsymbol{\xi}_{k}^{j}$ can be obtained as,

$$
w\left(\boldsymbol{\xi}_{k}^{j}\right)=\frac{w\left(\boldsymbol{\xi}_{k-1}^{j}\right) \mathcal{L}\left(\boldsymbol{\xi}_{k}^{j}\right)}{\sum_{l=1}^{N_{p}} w\left(\boldsymbol{\xi}_{k-1}^{l}\right) \mathcal{L}\left(\boldsymbol{\xi}_{k}^{l}\right)}
$$

The proposed algorithm is detailed in a pseudo-code in Algorithm 1 . 


\section{Numerical experiment}

The case study is built from a numerical FE model of a 3D two storey - one bay fixed base concrete frame structure consisting of sixteen members and 72 dofs (48 free dofs)(cf. Figure1). Each frame member is modelled with twelve dofs Euler Bernoulli beam element as detailed in Figure A.10 in Appendix A. The length and cross section for each member are assumed to be $3 m$ and $0.3 m \times 0.3 m$, respectively. The beam material is assumed to have a modulus of elasticity of $30 \mathrm{GPa}$, a modulus of rigidity of $10 \mathrm{GPa}$ and a density of $2500 \mathrm{~kg} / \mathrm{m}^{3}$. This frame is excited by the true recorded data of the bi-directional El-Centro earthquake ground motion (May 18, 1940 in CA, USA, direction North-South and East-West) (Data source: http: //peer.berkeley.edu/research/motions/). In addition, SWGN and/or non-stationary WGN (NSWGN) have been applied on all dof s throughout the simulation time, detailed later in the manuscript.

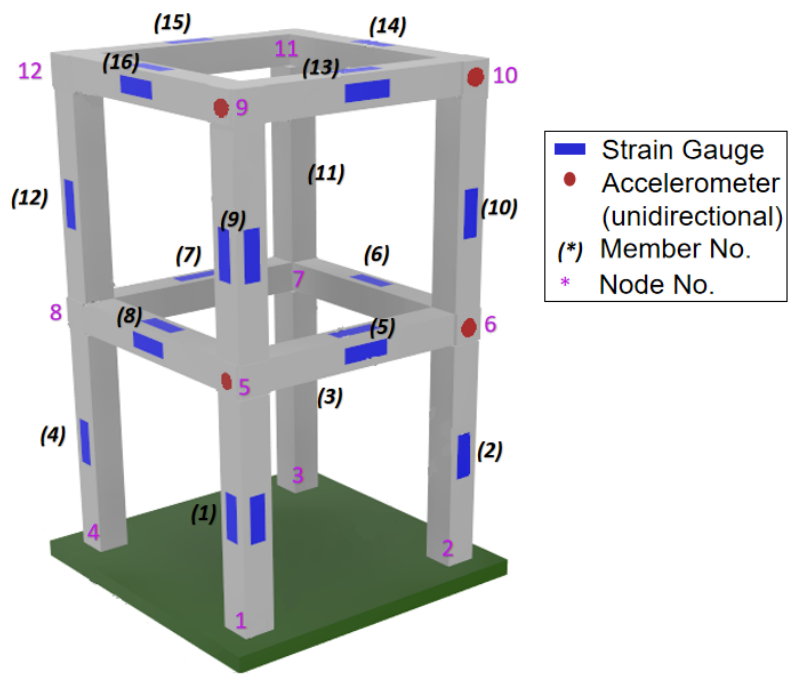

Figure 1: Schematic diagram of the sixteen member numerical frame

Strain measurements are collected from the strain gauges patched on to the top and the vertical external surfaces for the beams and the vertical external surfaces of the columns at their midpoints. In addition, horizontal accelerations are recorded at a set of nodes (cf. Figure 1). Various combinations of strain gauges and accelerometers have been tried, with a maximum of 32 strain gauges (two for each of 16 members) and 8 accelerometers. Responses are sampled at a fixed sampling frequency of $50 \mathrm{~Hz}$ for 61.44 seconds to collect the response time histories of length 3072. Noise contamination levels are defined in terms of signal-to-noise ratio (SNR) in which the noise power of an SWGN has been scaled with respect to the structural response against an SWGN vibration of variance 100 N. Various noise levels corresponding to different SNRs have been experimented with.

A numerical reduction in elasticity is considered as damage for the simulations. To maintain consistency, 
member 9 has been consistently assumed as damaged. A pool of 2000 particles are chosen for PF while 50 ensembles are chosen for EnKF. A better precision in estimation can be obtained with a bigger particle pool or a bigger set of ensembles, which however comes at a higher computational cost. The particle pool size and other tuning parameters relevant to the particle evolution have been standardized in [37, 38, 42. For EnKF, 50 ensembles have been found to be sufficient with no significant loss in precision. A Gaussian noise model, $\mathcal{N}(1,0.02)$, has been chosen as the initial distribution for the parameter particles with $\alpha$ chosen as 0.98 (cf. Equation (12). A lag has been introduced between the arrival of earthquake and the damage occurrence to mimic the reality.

The member health is quantified using HIs. $m$ HIs corresponding to $m$ individual members are estimated as individual elements of the $m \times 1$ order parameter vector $\boldsymbol{\theta}_{k}$. With a damage induced in the structure in terms of reduction in the initial elasticity $\mathbb{E}_{k}=\left\{\begin{array}{ll}E_{k}^{1} & E_{k}^{2} \cdots E_{k}^{m}\end{array}\right\}$ of its members, the effect of $\mathbf{H I s}$ as $\boldsymbol{\theta}_{k}$ on member elasticity can be defined as,

$$
\mathbb{E}_{k}^{d}\left(\boldsymbol{\theta}_{k}\right)=<\mathbb{E}_{k} \cdot \boldsymbol{\theta}_{k}>
$$

where $\mathbb{E}_{k}^{d}\left(\boldsymbol{\theta}_{k}\right)$ denotes the reduced elasticity of the potentially damaged members and $<\cdot>$ denotes the element-by-element multiplication operator. Since the structural stiffness $\mathbf{K}\left(\boldsymbol{\theta}_{k}\right)$ is a linear function of the member elasticity $\mathbb{E}_{k}^{d}\left(\boldsymbol{\theta}_{k}\right)$, HIs monitor the member health in terms of the ratio of the final to the initial member stiffness within a range of 0 to 1 , where 1 and 0 signify $0 \%$ and $100 \%$ damage levels. However, instead of a reduction in elasticity, any other definition for damage can be applied. The impact of HIs to the corresponding damaged stiffness is however required to be mapped. For instance, $\mathbf{H I}=0.25$ is roughly equivalent to a $40 \%$ loss in beam depth for a conventional rectangular beam, which is practical for a real world scenario.

\subsection{Scenario description}

A numerical investigation has been performed for undamaged, and therefore linear time invariant (LTI), systems with constant system matrices. Further, linear time varying (LTV) systems are also investigated for which the system matrices are varying because of the induced damage. These LTI and LTV systems are experimented in combination with three forcing types (SWGN, NSWGN and earthquake (EQ)) with both robust rIP-EnKF and non-robust IP-EnKF algorithms. This leads to 8 different scenarios (C1-C8) that have been tested to validate the proposed method's relative efficiency in estimating damage over the non-robust approach: i) LTI under SWGN and EQ (C1/2-LTI-EQ); ii) LTV under SWGN (C3/4-LTV-SWGN); iii) LTV under NSWGN (C5/6-LTV-NSWGN); and finally, iv) LTV under SWGN and EQ (C7/8-LTV-EQ), with odd and even numbered cases estimated with non-robust (NR) and robust (R) approaches, respectively. The assumed SWGN of variance $100 \mathrm{~N}$ mimics the ambient excitation of known statistics. NSWGN is modelled as two consecutive SWGNs (first one between 0-5 secs, second one between 5-62 secs) of variance $100 \mathrm{~N}$ and 
$1000 \mathrm{~N}$. The bi-directional El centro earthquake excitation is adopted as EQ. The corresponding seismic excitation is presented in Figure 2. SWGNs are applied from the initiation of the simulation while NSWGN and EQs are introduced at the fifth second followed by damages (if any) at the eighth second. For all the cases, the responses are contaminated with SWGN of different SNR levels. With rIP-EnKF and IP-EnKF algorithms, denoted as $\mathrm{R}$ and $\mathrm{NR}$, the case names in the figure are defined using the following formalism $<$ case number $>-<$ system $>$ - $<$ forcing $>$ - $<$ damage type $>$ - $<$ member number $>$ - $<$ estimation algorithm $>$.

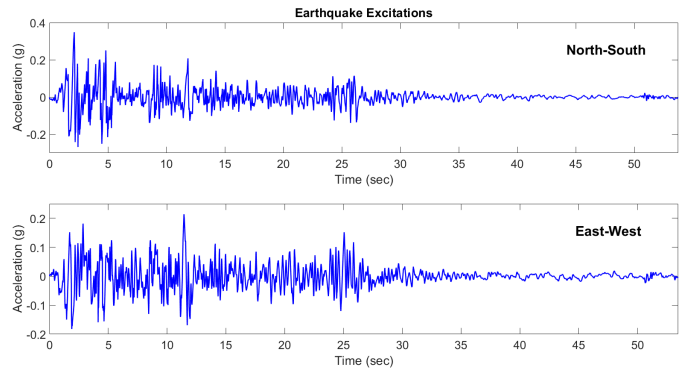

Figure 2: El centro seismic excitation in North-South (NS) and East-West (EW) direction

Further additional case studies (C9-C12) are performed to investigate the proposed method's sensitivity to measurement noises through a numerical Noise Sensitivity Test (NST). The following eight case studies (C13-C20) investigate the effect of two sensor combinations (SC1 and SC2) under four different noise contamination levels $(1 / 2 / 5 / 10 \% \mathrm{SNR})$. In this regard, additional results of ten numerical experiments (C-SC1 - C-SC10) corresponding to ten other sensor combinations (SC1-SC10) are presented to explicitly demonstrate the effect of sensor densities under different particle and ensemble pool sizes. Case studies C21-C22 demonstrate the capability of the proposed approach in detecting multiple damages while C-23 is included to illustrate the stability of the algorithm under a prolonged usage. A tabular description of each of the above mentioned scenarios has been given in Table 1 for better understanding. Each scenario has further been detailed in the subsequent sections.

\subsection{Robust vs Non-robust IP-EnKF approach}

The relative advantage of rIP-EnKF over the non-robust IP-EnKF is demonstrated through a comparative study presented in Figure 3 . For the sake of brevity, HI estimations are presented for two members only, one damaged (member 9) and one undamaged (member 1). Cases C1/2-LTI-EQ (Figure 3a) present that both IP-EnKF and rIP-ENKF perform equally good with the later being a little more stable. Further, in cases C3/4-LTV-SWGN (Figure 3b), better efficiency with rIP-EnKF becomes more evident. Till this phase, it is safe to conclude that neither of these two algorithms suffers from variations in the system matrices alone. Non-robust IP-EnKF starts performing poorly once the temporal variation is introduced in the input forcing in the case studies C5-LTV-NSWGN (cf. Figure3c) and C7-LTV-EQ (cf. Figure 3d). For C5-LTV-NSWGN, the solution is not even converging (cf. Figure 3c), while for C7-LTV-EQ, the convergence is unstable and 
Table 1: Details of numerical case studies

\begin{tabular}{|c|c|c|c|c|c|c|c|}
\hline Objective & Case name & Algorithm & System & Forces & $d_{l}$ & SNR & $N_{s}: N_{a}$ \\
\hline \multirow{8}{*}{$\begin{array}{l}\text { IP-EnKF } \\
\text { vs } \\
\text { rIPEnKF }\end{array}$} & C1-LTI-EQ & IP-EnKF & LTI & EQ+SWGN & $\mathrm{NA}$ & 1 & $32: 8$ \\
\hline & C2-LTI-EQ & rIP-EnKF & LTI & EQ+SWGN & NA & 1 & $32: 8$ \\
\hline & C3-LTV-SWGN & IP-EnKF & LTV & SWGN & 9 & 1 & $32: 8$ \\
\hline & C4-LTV-SWGN & rIP-EnKF & LTV & SWGN & 9 & 1 & $32: 8$ \\
\hline & C5-LTV-NSWGN & IP-EnKF & LTV & SWGN+NSWGN & 9 & 1 & $32: 8$ \\
\hline & C6-LTV-NSWGN & rIP-EnKF & LTV & SWGN+NSWGN & 9 & 1 & $32: 8$ \\
\hline & C7-LTV-EQ & IP-EnKF & LTV & EQ+SWGN & 9 & 1 & $32: 8$ \\
\hline & C8-LTV-EQ & rIP-EnKF & LTV & EQ+SWGN & 9 & 1 & $32: 8$ \\
\hline \multirow{4}{*}{$\begin{array}{c}\text { Noise } \\
\text { sensitivity } \\
\text { test }\end{array}$} & C9-NST & rIP-EnKF & LTV & EQ+SWGN & 9 & 1 & $32: 8$ \\
\hline & C10-NST & rIP-EnKF & LTV & $\mathrm{EQ}+\mathrm{SWGN}$ & 9 & 2 & $32: 8$ \\
\hline & C11-NST & rIP-EnKF & LTV & EQ+SWGN & 9 & 5 & $32: 8$ \\
\hline & C12-NST & rIP-EnKF & LTV & $\mathrm{EQ}+\mathrm{SWGN}$ & 9 & 10 & $32: 8$ \\
\hline \multirow{8}{*}{$\begin{array}{c}\text { Sensor } \\
\text { combination } \\
\text { test }\end{array}$} & $\mathrm{C} 13$ & rIP-EnKF & LTV & EQ+SWGN & 9 & 1 & $32: 4$ \\
\hline & C14 & rIP-EnKF & LTV & EQ+SWGN & 9 & 2 & $32: 4$ \\
\hline & C15 & rIP-EnKF & LTV & EQ+SWGN & 9 & 5 & $32: 4$ \\
\hline & C16 & rIP-EnKF & LTV & EQ+SWGN & 9 & 10 & $32: 4$ \\
\hline & C17 & rIP-EnKF & LTV & EQ+SWGN & 9 & 1 & $32: 8$ \\
\hline & C18 & rIP-EnKF & LTV & EQ+SWGN & 9 & 2 & $32: 8$ \\
\hline & C19 & rIP-EnKF & LTV & EQ+SWGN & 9 & 5 & $32: 8$ \\
\hline & $\mathrm{C} 20$ & rIP-EnKF & LTV & EQ+SWGN & 9 & 10 & $32: 8$ \\
\hline \multirow{2}{*}{$\begin{array}{l}\text { Double } \\
\text { damage }\end{array}$} & $\mathrm{C} 21$ & rIP-EnKF & LTV & $\mathrm{EQ}+\mathrm{SWGN}$ & $1 \& 5$ & 1 & $32: 8$ \\
\hline & $\mathrm{C} 22$ & rIP-EnKF & LTV & EQ+SWGN & $3 \& 7$ & 1 & $32: 8$ \\
\hline $\begin{array}{l}\text { Stability } \\
\text { check }\end{array}$ & $\mathrm{C} 23$ & rIP-EnKF & LTV & $\mathrm{EQ}+\mathrm{SWGN}$ & 9 & 1 & $32: 8$ \\
\hline
\end{tabular}

$N_{s}$ and $N_{a}$ denote the number of strain gauges and accelerometers, respectively. $d_{l}$ and SNR denote damaged element/s and signal-to-noise ratio for the given case.

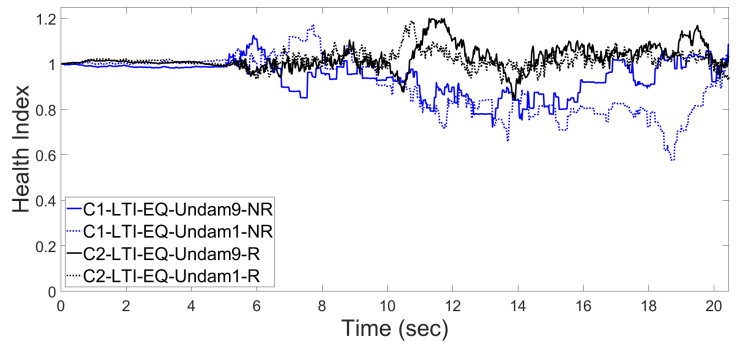

(a) LTI system with SWGN and EQ inputs

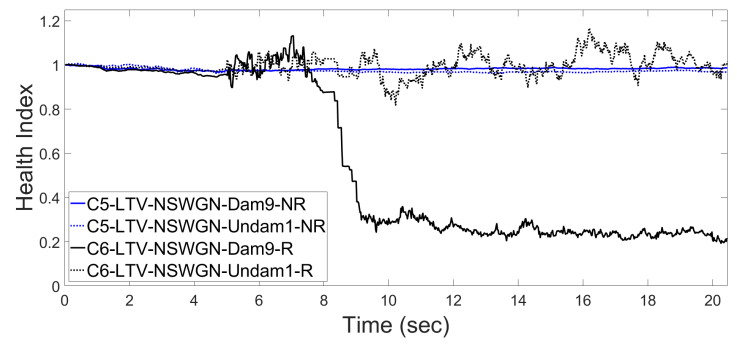

(c) LTV system with NSWGN input

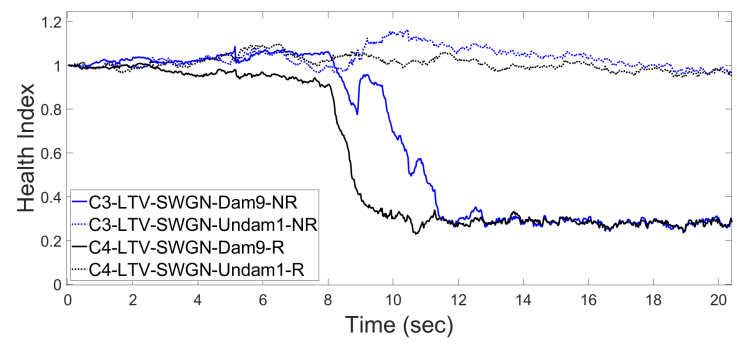

(b) LTV system with SWGN input

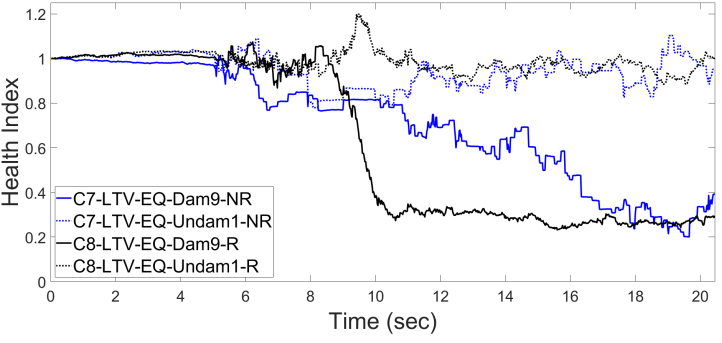

(d) LTV system with seismic excitation

Figure 3: Comparative study between IP-EnKF vs rIP-EnKF under different system (LTI/LTV) and input (Stationary/nonstationary/seismic) conditions

not prompt. It takes more samples than rIP-EnKF to approach the actual value of the parameter. The evolution is also not stable after convergence (cf. Figure $3 \mathrm{~d}$ ). Thus, the non-robust algorithm fails to handle this variation at the onset of change in the input statistics. Putting the same dataset through rIP-EnKF for C6-LTV-NSWGN (Figure 3c) and C8-LTV-EQ (Figure 3d), prompt and precise estimations are achieved. The estimation history for C8-LTV-EQ is further demonstrated in terms of particle dispersion and particle 
histogram in Figure 4
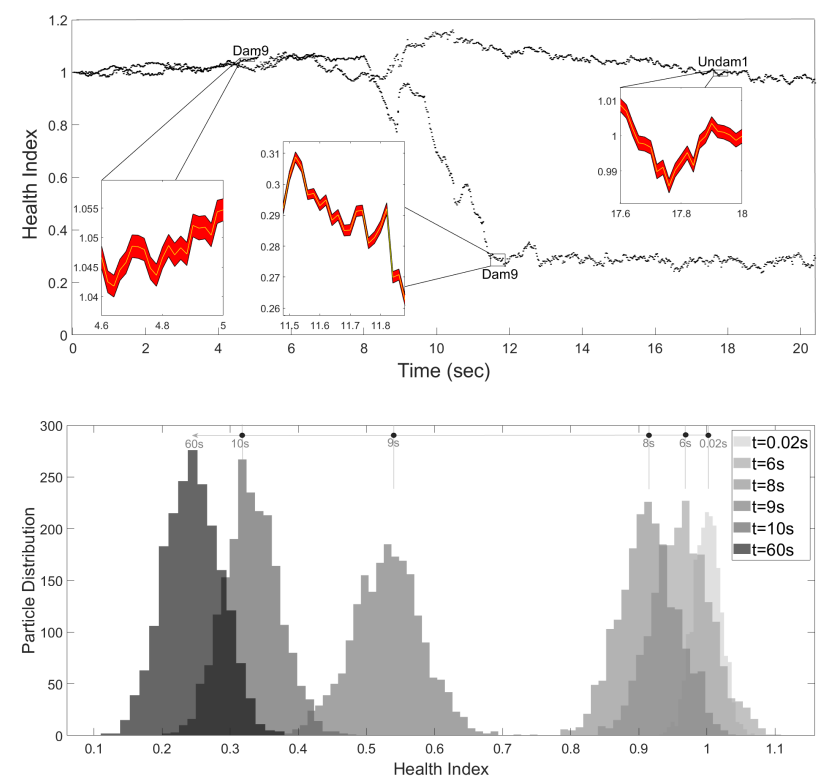

Figure 4: Temporal evolution of particles for damaged and undamaged members: Variation of standard deviation (top) and particle histogram (bottom)

\subsection{Robustness against measurement noise}

The proposed algorithm is tested for its robustness against measurement noise through four case studies corresponding to four increasing SNR levels (1/2/5/10\% SNR) while keeping the other parameters (damage location, extent and forcing) similar to that taken for the case study C8. The earthquake signal is introduced at the fifteenth second of simulation while damage is initiated at the twentieth second. Figure 5 presents the results of the noise sensitivity test for four case studies (C9-NST - C12-NST) corresponding to the four SNR levels adopted. As expected, with the increasing noise levels, the estimation promptness and precision gradually degrades (cf. Figure 5). Yet, the algorithm is observed to be efficient till SNR noise level of $5 \%$, beyond which (10\% SNR), some undamaged elements are falsely identified as slightly damaged. This illustrates the limiting noise contamination level for the algorithm to work precisely.

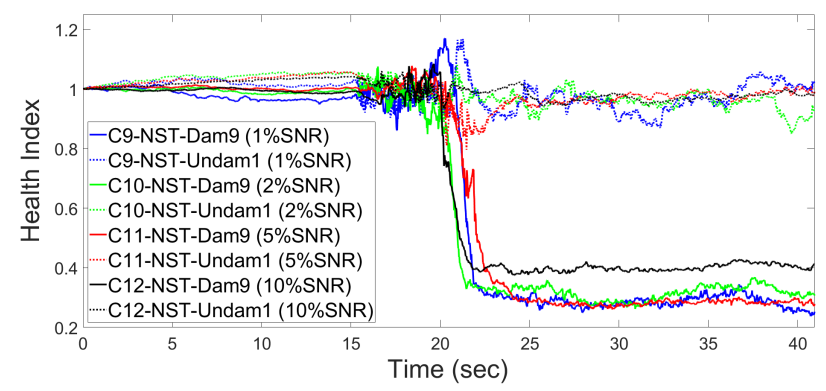

Figure 5: Measurement noise sensitivity of rIP-EnKF algorithm 


\subsection{Effect of measurement density and measurement type combinations}

From a theoretical point of view, it can be perceived that robustness against input forcing depends on the number of available linear measurement channels (acceleration in this case) while the detection precision is governed by the overall instrumentation density. Positioning of sensors with respect to damage locations, power in the recorded signal with respect to noise, size of particle and/or ensemble pools also play major roles in defining detection certainty. Thus, judging the detection ability of the proposed algorithm merely by sensor density may not be proper or practical. Yet a few test cases (C-SC1-C-SC10) are simulated for different sensor combinations and particle and ensemble pool sizes. The results are presented in Table 2

It is evident from Table 2, the proposed method efficiently estimates the damage location and severity even with reduced sensor densities. It can also be observed that a lack of sensors can be complimented with proper positioning of the sensors relative to the damage location and populating the ensemble and/or particle pools. Additionally, HI estimation for two case studies (Cases C13-SC11 - C20-SC12) corresponding to two sensor combinations (SC11: 32 strain gauges and 4 accelerometers and SC12: 32 strain gauges and 8 accelerometers) (other details are same as taken for C8) are presented in Figure 6a and 6b respectively. These case studies investigate the performance of rIP-EnKF under varying noise contamination levels $(1 / 2 / 5 / 10 \%$ SNR) under reduced instrumentation. Evidently, it can be observed that with reduced instrumentation, the algorithm's weakness to measurement noise increases.

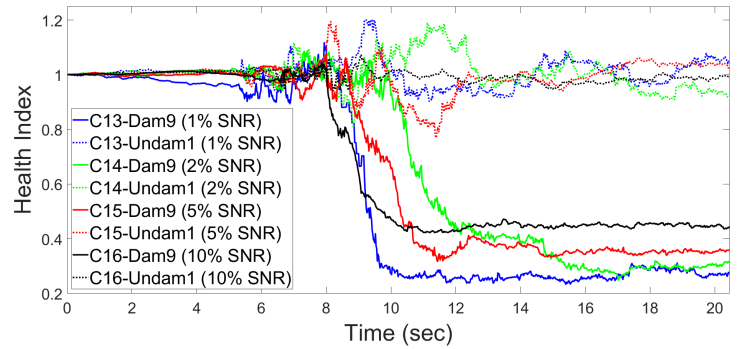

(a) Strain gauge vs Accelerometer ratio 32:4 - SC11

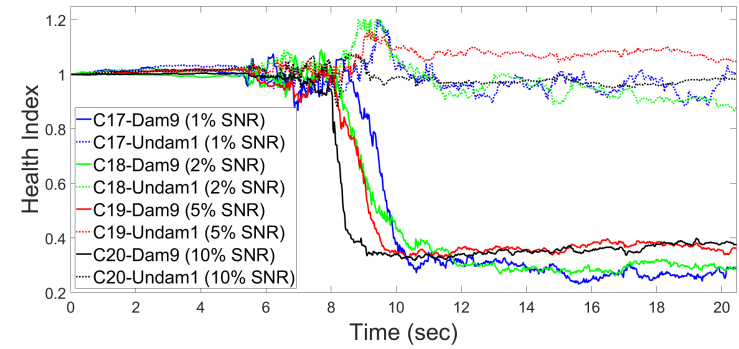

(b) Strain gauge vs Accelerometer ratio 32:8 - SC12

Figure 6: Performance of the algorithm under varying ratio of strain gauge and accelerometers

Table 2: Efficiency of varying ratio of strain gauge and accelerometers

\begin{tabular}{|c|c|c|c|c|c|c|}
\hline No. & $N_{s}$ & $N_{a}$ & $N_{e}$ & $N_{p}$ & $D^{11}$ & Acc \\
\hline C-SC1 & 16 & 4 & 50 & 2000 & $\checkmark$ & $99.4 \%$ \\
\hline C-SC2 & 16 & 2 & 50 & 2000 & $\checkmark$ & $99.4 \%$ \\
\hline C-SC3 & 16 & 1 & 50 & 2000 & $\checkmark$ & $98.8 \%$ \\
\hline C-SC4 & 8 & 4 & 50 & 2000 & $\checkmark$ & $73.3 \%$ \\
\hline C-SC5 & 8 & 2 & 75 & 2000 & $\checkmark$ & $94.4 \%$ \\
\hline
\end{tabular}

\begin{tabular}{|c|c|c|c|c|c|c|}
\hline No. & $N_{s}$ & $N_{a}$ & $N_{e}$ & $N_{p}$ & $D^{11}$ & $A c c$ \\
\hline C-SC6 & 2 & 1 & 200 & 2000 & $\checkmark$ & $94.4 \%$ \\
\hline C-SC7 & 4 & 1 & 100 & 2000 & $\checkmark$ & $88.9 \%$ \\
\hline C-SC8 & 4 & 2 & 100 & 2000 & $\checkmark$ & $94.4 \%$ \\
\hline C-SC9 & 4 & 1 & 75 & 2000 & $\checkmark$ & $88.9 \%$ \\
\hline C-SC10 & 4 & 2 & 100 & 3000 & $\checkmark$ & $96.1 \%$ \\
\hline
\end{tabular}

$N_{s}, N_{a}, N_{e}, N_{p}$ denote the number of strain gauges, accelerometers, ensembles and particles, respectively. $D^{11}$ and Acc denotes true detection and corresponding accuracies. 


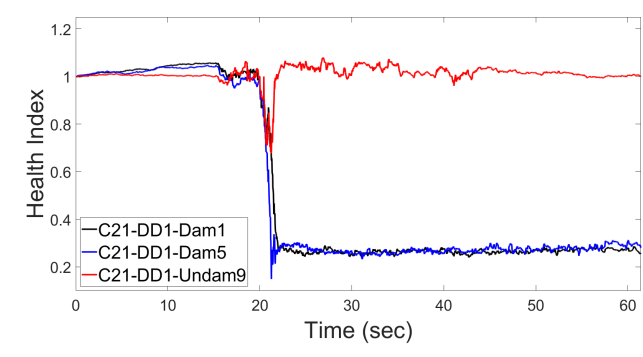

(a) Double damage detection study - DD1

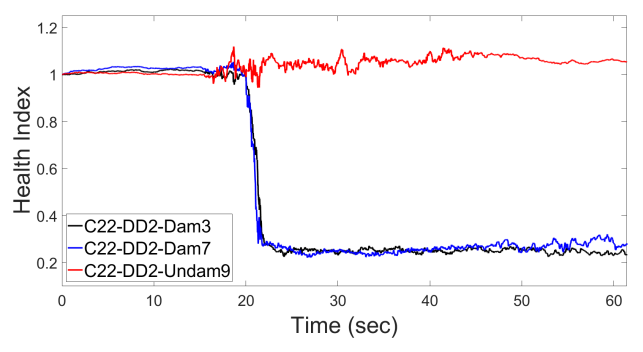

(b) Double damage detection study - DD2

Figure 7: Performance of the algorithm to detect multiple damage location and stability

\subsection{Detection of multiple damage scenario}

Multiple damage scenarios are also tested with the proposed algorithm through two case studies (C21C22). Figure 7 presents two such cases with damage at two locations - case study C21-DD1: with damages located at $1 \& 5$ (Figure 7a) and case study C22-DD2: with damages located at $3 \& 7$ (Figure 7b). A separate case to check the stability of the algorithm under prolonged usage is also undertaken in C23-SC. The forcing used in C9-NST - C12-NST is also used for the above mentioned cases. The algorithm performs promptly and precisely in detecting multiple damages (cf. Figure 7). Case study, C23-SC, tests the proposed algorithm's stability for a period of 388 seconds against possible error accumulation or divergence. The algorithm is observed to be stable for the mentioned duration showing no trend or significant turbulence (cf. Figure 8).

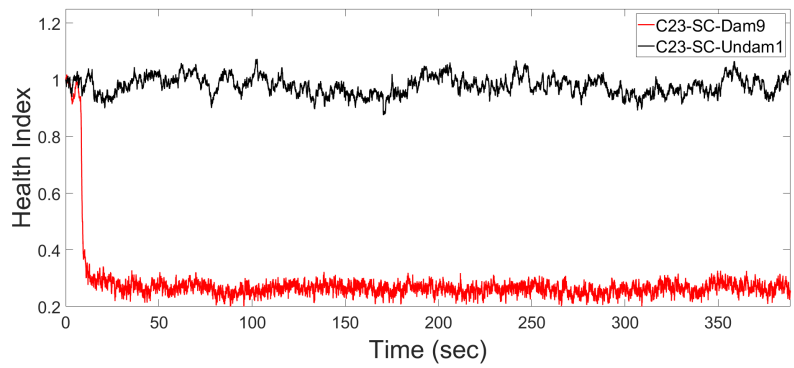

Figure 8: Stability check of the algorithm

The limiting value of damage that can be tracked with the proposed algorithm is investigated next. The results are presented in Figure 9 corresponding to four different damage levels (75\%, 30\%, $20 \%$ and 10\%). It has been observed that rIP-EnKF is consistent in detecting the damage occurrence even for $10 \%$ damage level $(\mathbf{H I}=0.9)$. The precise estimation of the damage extent should however be limited to $20 \%$ damage levels corresponding to $\mathbf{H I}=0.80$.

Overall, the proposed algorithm took about 5448 seconds of $c p u$ time to process a 1024 long time series, with 2000 particles and 50 ensembles for any case study discussed in this article. The employed computation system is equipped with Intel(R) Xeon(R) Silver $4210 \mathrm{CPU} @ 2.2 \mathrm{GHz} 2.19 \mathrm{GHz}$ (2 processors and 20 physical cores with multi threading capability) with 64 GB RAM. 


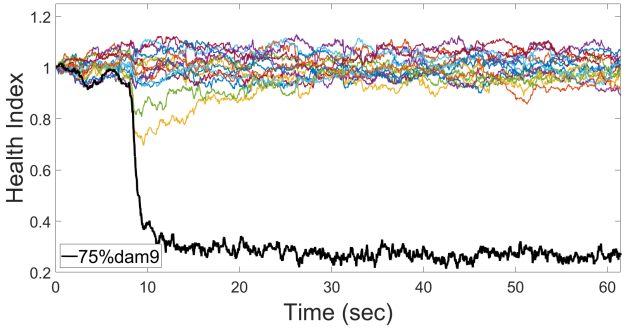

(a) Damage extent study - $75 \%$

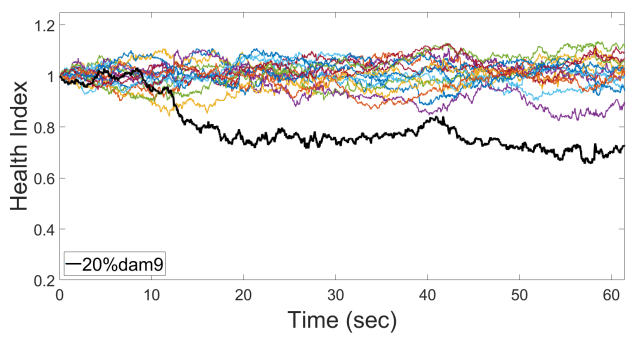

(c) Damage extent study - $20 \%$

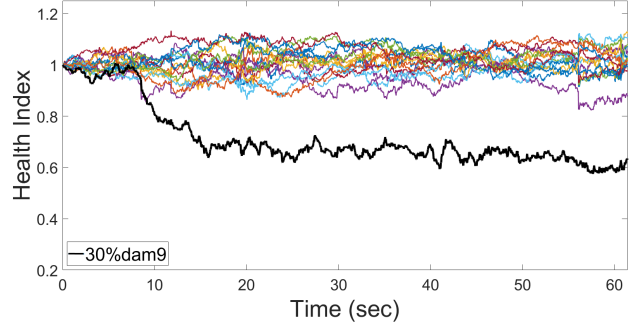

(b) Damage extent study - $30 \%$

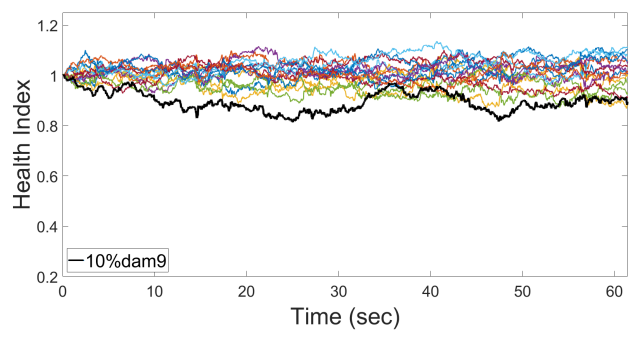

(d) Damage extent study - $10 \%$

Figure 9: Performance of the algorithm to detect various damage levels

\section{Conclusions}

This paper has presented a Bayesian filtering-based structural health monitoring strategy robust to unknown and arbitrary input forcing using a mix of linear (acceleration) and non-linear (strain) sensor measurements. The considered framework leads to a non-linear estimation problem beyond the capability of classical Kalman filtering. The proposed algorithm is thus based on an interacting filtering strategy coupling Ensemble Kalman filters (EnKF), to track the evolution of the system states, with a Particle filter (PF), to track the changes in the system parameters due to damage. The input robustness is achieved through an output injection technique embedded within the EnKF formalism. The efficacy, robustness, stability and sensitivity of the proposed approach is validated numerically.

Funding acknowledgement: This study is partially funded by DST-SERB, New Delhi, India, Grant file no. ECR/2018/001464.

\section{References}

[1] Astroza, R., Ebrahimian, H., Li, Y., Conte, J.P., 2017. Bayesian nonlinear structural fe model and seismic input identification for damage assessment of civil structures. Mechanical Systems and Signal Processing 93, 661-687.

[2] Azam, S.E., Bagherinia, M., Mariani, S., 2012a. Stochastic system identification via particle and sigma-point kalman filtering. Scientia Iranica 19, 982-991.

[3] Azam, S.E., Chatzi, E., Papadimitriou, C., 2015. A dual kalman filter approach for state estimation via output-only acceleration measurements. Mechanical Systems and Signal Processing 60, 866-886.

[4] Azam, S.E., Ghisi, A., Mariani, S., 2012b. Parallelized sigma-point kalman filtering for structural dynamics. Computers \& Structures 92, 193-205.

[5] Álvarez Briceño, R., de Oliveira, L.P., 2020. Combining strain and acceleration measurements for random force estimation via kalman filtering on a cantilevered structure. Journal of Sound and Vibration 469, 115112 
[6] Castiglione, J., Astroza, R., Azam, S.E., Linzell, D., 2020. Auto-regressive model based input and parameter estimation for nonlinear finite element models. Mechanical Systems and Signal Processing 143, 106779

[7] Chatzi, E.N., Smyth, A.W., 2009. The unscented kalman filter and particle filter methods for nonlinear structural system identification with non-collocated heterogeneous sensing. Structural Control and Health Monitoring 16, 99-123.

[8] Chen, Z., et al., 2003. Bayesian filtering: From kalman filters to particle filters, and beyond. Statistics 182, 1-69.

[9] Ching, J., Beck, J.L., Porter, K.A., 2006. Bayesian state and parameter estimation of uncertain dynamical systems. Probabilistic engineering mechanics 21, 81-96.

[10] Corigliano, A., Dossi, M., Mariani, S., 2015. Model order reduction and domain decomposition strategies for the solution of the dynamic elastic-plastic structural problem. Computer Methods in Applied Mechanics and Engineering 290, $127-155$.

[11] Corigliano, A., Mariani, S., 2004. Parameter identification in explicit structural dynamics: performance of the extended kalman filter. Computer Methods in Applied Mechanics and Engineering 193, 3807-3835.

[12] Dertimanis, V.K., Chatzi, E., Azam, S.E., Papadimitriou, C., 2019. Input-state-parameter estimation of structural systems from limited output information. Mechanical Systems and Signal Processing 126, 711-746.

[13] Doucet, A., De Freitas, N., Murphy, K., Russell, S., 2000. Rao-blackwellised particle filtering for dynamic bayesian networks, in: Proceedings of the Sixteenth conference on Uncertainty in artificial intelligence, Morgan Kaufmann Publishers Inc.. pp. 176-183.

[14] Evensen, G., 2003. The ensemble kalman filter: Theoretical formulation and practical implementation. Ocean dynamics $53,343-367$.

[15] Ge, M., Kerrigan, E.C., 2014. Noise covariance estimation for time-varying and nonlinear systems. IFAC Proceedings Volumes 47, 9545 - 9550. 19th IFAC World Congress.

[16] Gillijns, S., De Moor, B., 2007a. Unbiased minimum-variance input and state estimation for linear discrete-time systems. Automatica 43, 111-116.

[17] Gillijns, S., De Moor, B., 2007b. Unbiased minimum-variance input and state estimation for linear discrete-time systems with direct feedthrough. Automatica 43, 934-937.

[18] Gordon, N.J., Salmond, D.J., Smith, A.F., 1993. Novel approach to nonlinear/non-gaussian bayesian state estimation, in: IEE proceedings F (radar and signal processing), IET. pp. 107-113.

[19] Hommels, A., Molenkamp, F., Heemink, A., Nguyen, B., 2005. Inverse analysis of an embankment on soft clay using the ensemble kalman filter, in: Proc. of the 10th Int. Conf. on Civil, Structural and Env. Eng. Computing, Civil-Comp Press, Stirling, United Kingdom, paper.

[20] Hommels, A., Murakami, A., Nishimura, S.I., 2009. A comparison of the ensemble kalman filter with the unscented kalman filter: application to the construction of a road embankment. Geotechniek 13, 52.

[21] Hoshiya, M., Saito, E., 1984. Structural identification by extended kalman filter. Journal of engineering mechanics 110, 1757-1770.

[22] Hsieh, C.S., 2000. Robust two-stage kalman filters for systems with unknown inputs. IEEE Transactions on Automatic Control 45, 2374-2378.

[23] Julier, S.J., Uhlmann, J.K., 1997. New extension of the kalman filter to nonlinear systems, in: Signal processing, sensor fusion, and target recognition VI, International Society for Optics and Photonics. pp. 182-194.

[24] Kitanidis, P.K., 1987. Unbiased minimum-variance linear state estimation. Automatica 23, 775-778.

[25] Li, X.R., Jilkov, V.P., 2001. Survey of maneuvering target tracking: Iii. measurement models, in: Signal and Data Processing of Small Targets 2001, International Society for Optics and Photonics. pp. 423-446.

[26] Lourens, E., Papadimitriou, C., Gillijns, S., Reynders, E., De Roeck, G., Lombaert, G., 2012a. Joint input-response estimation for structural systems based on reduced-order models and vibration data from a limited number of sensors. Mechanical Systems and Signal Processing 29, 310-327.

[27] Lourens, E., Reynders, E., De Roeck, G., Degrande, G., Lombaert, G., 2012b. An augmented kalman filter for force identification in structural dynamics. Mechanical Systems and Signal Processing 27, 446-460.

[28] Maes, K., Gillijns, S., Lombaert, G., 2018. A smoothing algorithm for joint input-state estimation in structural dynamics. Mechanical Systems and Signal Processing 98, 292-309.

[29] Maes, K., Karlsson, F., Lombaert, G., 2019. Tracking of inputs, states and parameters of linear structural dynamic systems. Mechanical Systems and Signal Processing 130, 755-775.

[30] Mariani, S., Ghisi, A., 2007. Unscented kalman filtering for nonlinear structural dynamics. Nonlinear Dynamics 49, $131-150$.

[31] Mercère, G., Bako, L., Lecœuche, S., 2008. Propagator-based methods for recursive subspace model identification. Signal Processing 88, 468-491.

[32] Morelande, M.R., Garcia-Fernandez, A.F., 2013. Analysis of kalman filter approximations for nonlinear measurements. IEEE Transactions on Signal Processing 61, 5477-5484.

[33] Orderud, F., 2005. Comparison of kalman filter estimation approaches for state space models with nonlinear measurements, in: Proc. of Scandinavian Conference on Simulation and Modeling, pp. 1-8.

[34] Rogers, T., Worden, K., Cross, E., 2020. On the application of gaussian process latent force models for joint input-stateparameter estimation: With a view to bayesian operational identification. Mechanical Systems and Signal Processing 140, 106580 .

[35] Sen, S., Bhattacharya, B., 2016. Progressive damage identification using dual extended kalman filter. Acta Mechanica 227, 2099-2109.

[36] Sen, S., Bhattacharya, B., 2017. Online structural damage identification technique using constrained dual extended kalman filter. Structural Control and Health Monitoring 24, e1961.

[37] Sen, S., Crinìere, A., Mevel, L., Cérou, F., Dumoulin, J., 2018a. Correntropy based ipkf filter for parameter estimation in 
presence of non-stationary noise process. IFAC-PapersOnLine 51, 420-427.

[38] Sen, S., Crinière, A., Mevel, L., Cérou, F., Dumoulin, J., 2018b. Seismic-induced damage detection through parallel force and parameter estimation using an improved interacting particle-kalman filter. Mechanical Systems and Signal Processing 110, 231-247.

[39] Snyder, C., Bengtsson, T., Bickel, P., Anderson, J., 2008. Obstacles to high-dimensional particle filtering. Monthly Weather Review 136, 4629-4640.

[40] Van Der Merwe, R., Wan, E.A., 2001. The square-root unscented kalman filter for state and parameter-estimation, in: 2001 IEEE international conference on acoustics, speech, and signal processing. Proceedings (Cat. No. 01CH37221), IEEE. pp. 3461-3464.

[41] Welch, G., Bishop, G., et al., 1995. An introduction to the kalman filter.

[42] Zghal, M., Mevel, L., Del Moral, P., 2014. Modal parameter estimation using interacting kalman filter. Mechanical Systems and Signal Processing 47, 139-150.

[43] Zhang, Q., Zhang, L., 2018. State estimation for stochastic time varying systems with disturbance rejection. IFACPapersOnLine 51, 55-59.

[44] Zhao, Z., Li, T.R., Jilkov, V.P., 2004. Best linear unbiased filtering with nonlinear measurements for target tracking. IEEE Transactions on Aerospace and electronic systems 40, 1324-1336.

\section{Appendix A. Strain-diaplcement function for Euler-bernoulli beam}

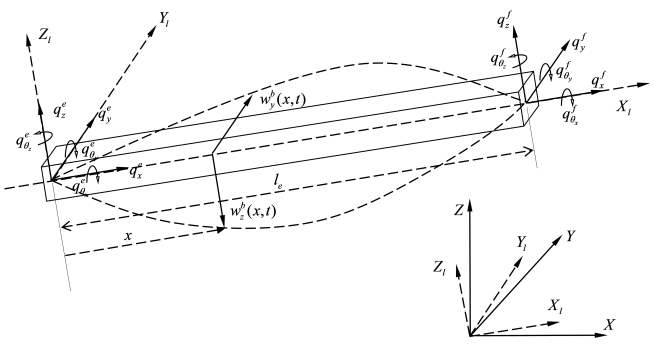

Figure A.10: Assumed Euler-Bernoulli Beam element with local and global degrees of freedom

A Strain-displacement mapping using FE model is performed using 3D Euler-Bernoulli beam elements schematically drawn in Figure A.10. The transverse deflection, $w(x, t)$ at a distance $x$ along beam orientation (i.e., $X_{l}$ ), with its components, $w_{y}(x, t)$ and $w_{z}(x, t)$ along $Y_{l}$ and $Z_{l}$, can be defined as the interpolation of the nodal displacements as,

$$
w(x, t)=\left[\begin{array}{l}
w^{b}(x, t) \\
w^{a}(x, t)
\end{array}\right]=\left[\begin{array}{l}
w_{y}^{b}(x, t) \\
w_{z}^{b}(x, t)
\end{array}\right]+\left[\begin{array}{l}
w_{y}^{a}(x, t) \\
w_{z}^{a}(x, t)
\end{array}\right]=\boldsymbol{\psi}(x) \boldsymbol{q}^{l}(t)
$$

$\boldsymbol{q}^{l}(t)=\left[\begin{array}{llllllllllll}q_{x}^{e} & q_{y}^{e} & q_{z}^{e} & q_{\theta_{x}}^{e} & q_{\theta_{y}}^{e} & q_{\theta_{z}}^{e} & q_{x}^{f} & q_{y}^{f} & q_{z}^{f} & q_{\theta_{x}}^{f} & q_{\theta_{y}}^{f} & q_{\theta_{z}}^{f}\end{array}\right]^{T}$ can be retrieved from the nodal displacements, $\mathbf{q}(t)$, defined in Global Coordinate System (GCS) through coordinate transformation as $\boldsymbol{q}^{l}(t)=$ $\mathbf{T q}(t)$ where $\mathbf{T}$ is the coordinate transformation matrix. $\boldsymbol{\psi}(x)$ is the interpolation function constituted with the associated shape functions. Similar to displacement, the slope $\phi^{b}(x, t)$ and curvature $\kappa^{b}(x, t)$ at $x$ at time $t$ due to bending can be obtained as, $\phi^{b}(x, t)=\boldsymbol{\psi}^{b}(x)^{\prime} \boldsymbol{q}^{l}(t)$ and $\kappa^{b}(x, t)=\left(1+\phi^{b}(x, t)^{2}\right)^{\frac{3}{2}}\left\{\boldsymbol{\psi}^{b}(x)^{\prime \prime} \boldsymbol{q}^{l}(t)\right\}^{-1}$. The curvature, measured through longitudinal strains, $\left.\boldsymbol{\varepsilon}^{x x}(x, t)\right)$, is eventually a function of $\boldsymbol{q}^{l}(t)$ or $\boldsymbol{q}(t)$. $\mathbf{q}(t)$ being a subset of the state variable $\mathbf{x}(t)$ yields the non-linear mapping $\varepsilon^{x x}(x, t)=f(\mathbf{x}(t)$ to describe strain-displacement relationship. 